\title{
Hypoarginaemia among Pre- School Children of African Descent with Plasmodium Falciparum Malaria
}

\author{
Kabiru $\mathbf{M}^{1}$, Erhabor $\mathbf{0}^{1 *}$, Isaac $\mathrm{Z}^{1}$, Jiya $\mathrm{NM}^{2}$, Knox Van $\mathrm{D}^{3}$, \\ Abdulrahaman $\mathrm{Y}^{1}$ and Egenti $\mathrm{N}^{4}$ \\ ${ }^{1}$ Department of Haematology and Blood Transfusion Science, Usmanu Danfodiyo \\ University, Nigeria \\ ${ }^{2}$ Department of Paediatrics, Usmanu Danfodiyo University, Nigeria \\ ${ }^{3}$ Department of Biochemistry, West Virginia University, USA \\ ${ }^{4}$ Department of Community Medicine University of Abuja, Nigeria
}

*Corresponding author: Osaro Erhabor, Department of Haematology and Blood Transfusion Science, Faculty of Medical Laboratory Science, Usmanu Danfodiyo University, Sokoto, Nigeria, E-mail: n_osaro@yahoo.com

\section{Abstract}

Malaria is a public health problem particularly in the tropical areas of the world affecting about 247 million people each year and often resulting in nearly a million deaths. It is the leading cause of under-five hospital admission in endemic countries. L-Arginine is a precursor for nitric oxide synthesis. In this study, the level of l-arginine was investigated among pre-school children with malaria presenting to the Pediatric Outpatient Department of Usmanu Danfodiyo University Teaching Hospital Sokoto, Nigeria. A total of 90 pre- school children with malaria aged 3-5 year with mean age of $4.01 \pm 0.87$ and 50 non- parasitized age and gender- matched pre-school non-hospitalized children (control) were recruited for this study. There was a statistical significance difference in the serum L-arginine level among children with malaria compared to non-parasitized children $(\mathrm{p}=0.001)$. There was a statistical significance difference in serum L-arginine level of children with severe malaria compare to uncomplicated malaria children ( $\mathrm{p}=$ 0.000). There were no age and gender -related differences in the serum L-arginine levels among pre-school children with malaria ( $\mathrm{p}=0.11$ and 0.93 respectively). There was no significance difference in the serum L-arginine level of the malaria parasitized children based on ethnicity $(\mathrm{p}=0.59)$. This study shows that P. falciparum malaria is associated with hypoarginaemia among preschool children of African descent. It is recommended serum L-arginine level be determined for malaria parasitized children. It may be necessary to routinely provide L-arginine supplements early during malaria infection as an adjuvant therapy with chemotherapeutic agents against malaria.

Keywords: Hypoarginaemia; Pre- School Children; African; Plasmodium falciparum; Malaria

\section{Introduction}

Malaria is the most important tropical parasitic disease affecting about 247 million people each year, resulting in nearly a million deaths, mostly children under the age of five years [1]. Nearly $90 \%$ of these 
deaths occur in Africa, South of the Sahara where a child dies every 30 seconds [2]. Pregnant women and their unborn children are also particularly vulnerable to malaria, which is a major cause of prenatal mortality, low birth weight and maternal anaemia. It accounts for $40 \%$ of public health expenditure, $30-50 \%$ of inpatient admissions and up to $50 \%$ of out-patient visits in areas with high malaria transmission [3]. NO is produced by various group of enzymes termed as nitric oxide syntheses (NOS) which are present in the body $[4,5]$. The synthesis of NO takes place by the conversion of L-arginine to L-citrulline, the reaction being catalyzed by nitric oxide synthases (NOS) [6].

Malaria is endemic throughout Nigeria with seasonal variation in different geographic zones of the country. More than $90 \%$ of the total population is at risk of malaria and at least $50 \%$ of the population suffers from at least one episode of malaria each year. Beyond the impact on children and pregnant women, it affects the general population $[7,8]$. Despite the use of fast-acting antimalarial such as artemisinins, case mortality rates in severe malaria remain high, underlining the significance of adjunctive therapies [9]. Some common manifestations of severe malaria include low arginine (hypoargininemia) and endothelial dysfunction [10]. As L-Arginine is the substrate of NO synthase (NOS) for the production of NO, hypoargininemia can lead to impaired systemic NO production [11]. The most common life-threatening clinical syndromes associated with P. falciparum infection in children are cerebral malaria (CM), respiratory distress with metabolic acidosis, and severe malarial anaemia [12]. Critical pathogenic mechanisms in these severe malaria syndromes represent potential targets for adjunctive therapies, including nitric oxide. The pathogenesis of CM is incompletely understood but may be due to one or more of the following mechanisms: sequestration of parasitized erythrocytes within the cerebral microvasculature $[13,14]$, reduction in microvascular flow [15], metabolic alterations including hypoglycemia and hypoxia, host inflammatory response $[16,17]$, blood-brain barrier dysfunction [18,19] and cerebral oedema [20,21]. Among African children with severe malaria, lactic acidosis secondary to impaired tissue perfusion appears to explain most cases of respiratory distress, characterized by deep respirations [22]. Respiratory distress therefore represents a manifestation of decompensated shock, frequently associated with multi-system organ failure and widespread endothelial dysfunction. Severe malarial anaemia is caused by both increased destruction of parasitized and non-parasitized erythrocytes, as well as impaired haematopoiesis [23]. There is a need to determine the levels of L-arginine in uncomplicated malaria to reduce the unacceptably high case-fatality of severe malaria (SM). Irrespective of its cause, hypoargininemia likely limits NO production and may be associated with death in cerebral malaria [24]. There are no NO-arginine-malaria studies in Nigeria and the results of arginine trials in related diseases suggest that arginine may have potential for therapeutic use in malaria. L-arginine is commercially available as an offpatent product for human use, is inexpensive, and has been widely used worldwide with little toxicity despite supra-physiological intravenous dosing as a diagnostic pituitary function test and administration in other human diseases [25]. There is paucity of data on serum L-arginine levels among malaria parasitized Nigerian children. Findings from this study may potentially be used to justify the need to routinely determine the level of serum L-arginine among pre-school children with malaria. It may also help to justify the need to prescribe concomitant $\mathrm{L}$-arginine supplements with antimalarials in the treatment of pre-school children with malaria in Sokoto State in particular and Nigeria in general. The aim of the study is to determine the serum levels of 1 arginine among children with malaria in UDUTH Sokoto, Nigeria.

\section{Materials and Methods}

\section{Study Design}

The study is a quantitative study to determine the levels of serum l-arginine among pre-school children with malaria in UDUTH, Sokoto Nigeria. This study investigated 140 individuals; 90 Plasmodium parasitized pre-school children (subjects) and 50 nonparasitized non-hospitalized children (controls).

\section{Study Site}

The study was conducted in Usmanu Danfodiyo University Teaching Hospital (UDUTH) at the Paediatric Outpatient Department in collaboration with the Department of Haematology and Blood Transfusion in the Faculty of Medical Laboratory Science in Usmanu Danfodiyo University Sokoto, Nigeria. Sokoto is the capital city of Sokoto State. It is located in the extreme North-Western part of Nigeria. Sokoto metropolis is estimated to have a population of 427,760 people [26] and by the virtue of its origin, the state comprises mostly of Hausa/Fulani and other groups such as Gobirawa, Zabarmawa, Kabawa, Adarawa, Arawa, Nupe, Yoruba, Ibos and others. Occupation of the inhabitants include trading, commerce, with a reasonable proportion of the population working in private and public sectors [27]. The Sokoto township is in the dry Sahel surrounded by sandy terrain and isolated hills [28]. The city is a major commerce center in leather crafts and agricultural products [27]. 


\section{Ethical Clearance}

Ethical approval for this research was sought from the Ethics Committee of Usmanu Danfodiyo University Teaching Hospital Sokoto. Verbal informed written consent was obtained from parents or guardian of all study participants after counseling.

\section{Eligibility Criteria}

Eligibility criteria include age (3-5 years), confirmed malaria infection at presentation at the paediatric unit and willingness of the parents or guardian to offer a verbal informed consent for their ward to participate in this study.

\section{Exclusion Criteria}

The following children who did not meet the inclusion criteria were excluded from participating as subjects in the study; all children $<3$ and $>5$ years, nonhospitalized malaria negative children and children whose parents have not given a verbal informed consent for their ward to participate as subjects in the study.

\section{Sampling Method}

Samples was collected from consecutively recruited children attending pediatric unit of Usmanu Danfodiyo University Teaching Hospital Sokoto who parents gave a verbal informed consent for their wards to participate in the study.

\section{Specimen Collection and Preparation}

Five milliliters of blood were obtained from the subjects and control participants by venipuncture into EDTA anticoagulated tubes (2 milliliters) and plain tubes (3 milliliters). The EDTA sample was used for blood smears (thick blood films). The thick blood films were stained by Giemsa's stain. The number of asexual parasites among the parasitized subjects was counted and parasite densities computed while the thin blood film was stained using Leishman stain and used for speciation. The sample collected in the plain tube was allowed to clot naturally and centrifuged to obtain serum. L-arginine levels in the serum were determined using enzyme-linked immunosorbent assay (ELISA) kits (Immunodiagnostik, Germany).

\section{Parasitological Technique (Malaria Parasite)}

Thick blood film

1. Thick blood films were made on clean grease-free slides.

2. The slides were stained using Giemsa staining solution.

3. The stain was flushed off using distilled water
4. The slide was then allowed to air dry

5. The stained films were then examined microscopically using $100 \mathrm{x}$ objectives to count the parasites.

\section{Principle of the Test}

The l-arginine assay is based on the method of competitive enzyme linked immunoassays. The sample preparation includes the addition of a derivatization reagent for L-arginine derivatization. Afterwards, the treated samples and the polyclonal L-arginine antiserum are incubated in wells of a microtiter plate coated with L-arginine-derivative (tracer). During the incubation period, the target L-arginine in the sample competes with the tracer immobilized on the wall of the microtiter wells for the binding of the polyclonal antibodies. The L-arginine in the sample displaces the antibodies out of the binding to the tracer. Therefore, the concentration of the tracer bound antibody is inverse proportional to the L-arginine concentration in the sample. During the second incubation step, a peroxidase-conjugated antibody is added to each microtiter well to detect the anti-L-arginine antibodies. After washing away the unbound components tetramethylbenzidine (TMB) was added as a peroxidase substrate. Finally, the enzymatic reaction was terminated by an acidic stop solution. The colour changes from blue to yellow, and the absorbance is measured in a photometer at $450 \mathrm{~nm}$. The intensity of the yellow colour is inverse proportional to the Larginine concentration in the sample. This means that a high L-arginine concentration in the sample reduces the concentration of tracer-bound antibodies and lowers the photometric signal.

\section{Test Procedure}

a) Each well was washed 5 times by dispensing $250 \mu \mathrm{l}$ of diluted wash buffer into each well. After the final washing step, the inverted microtiter plate (PLATE) was firmly tapped on absorbent paper to remove excess solution.

b) $150 \mu \mathrm{l}$ of diluted anti-L-arginine antibody (AB) was added into each well, and the plate was covered tightly.

c) The plate was incubated overnight for 15-20 hours at $2-8^{\circ} \mathrm{C}$.

d) The contents of each well were aspirated. Each well was washed 5 times by dispensing $250 \mu \mathrm{l}$ of diluted wash buffer into each well.

e) $200 \mu$ diluted POD antibody (2. AB) was added into each well.

f) The plate was covered tightly and incubated for 1 hour at room temperature $\left(18-26^{\circ} \mathrm{C}\right)$ on a horizontal shaker (180-240 rpm). 
g) The contents of each well were aspirated. Each well was then washed 5 times by dispensing $250 \mu \mathrm{l}$ of diluted wash buffer into each well.

h) $200 \mu \mathrm{l}$ of tetramethylbenzidine (TMB) substrate (SUB) was added into each well.

i) The plate was incubated for $12-16 \mathrm{~min}$ at room temperature $\left(18-26^{\circ} \mathrm{C}\right)$ in the dark.

j) $100 \mu \mathrm{l}$ of stop solution (STOP) was added into each well, and mixed thoroughly.

k) The absorption was determined immediately with an ELISA reader at $450 \mathrm{~nm}$.

\section{Measurement}

The ELISA micro well plate reader was adjusted to $450 \mathrm{~nm}$ with graph, six standard and two controls as provided in l-arginine ELISA kit. The absorbance of all well at $450 \mathrm{~nm}$ was read and recorded as the absorbance values for each standard, control and sample.

\section{Interpretation of Results}

a. Samples are considered high if the absorbance value is higher than $134.9 \mu \mathrm{mol} / \mathrm{l}$.

b. Samples are considered normal if the absorbance value is within $33.7-134.9 \mu \mathrm{mol} / \mathrm{l}$.

c. Samples are considered low if the absorbance value is lower than $33.7 \mu \mathrm{mol} / \mathrm{l}$.

\section{Statistical Analysis}

The data collected was descriptive and analysed using statistical software (SPSS version 20). Results were expressed as mean and standard deviation. Differences in values were determined and compared statistically using analysis of variance and student's ttest. A p-value of $\leq 0.05$ was considered as significant in all statistical comparisons.

\section{Result}

A total of 90 pre-school children with malaria aged 35 years with mean age of $4.01 \pm 0.87$ years and 50 age and gender matched non- parasitized pre-school children (control) were recruited for this study). Table 1 shows the results of mean score and serum L-arginine levels among pre-school children with malaria and nonmalaria parasitized controls. It was observed that the 1arginine among children with malaria (36.29 \pm $48.15 \mu \mathrm{mol} / \mathrm{l}$ ) was significantly lower compared to nonparasitized controls $(60.95 \pm 23.72 \mu \mathrm{mol} / \mathrm{l})(\mathrm{p}=0.001)$.

Table 1 Results of mean score and L-argin levels. Table 2 serum L-arginine levels and malaria. Table 3 serum levels by gender, Table 4 serum levels by clinically. Table 5 adjusted as above

\begin{tabular}{|c|c|c|c|c|c|c|c|c|}
\hline Parameter & Group & $\mathbf{N}$ & Mean \pm SD & $\begin{array}{c}\text { Std. Error } \\
\text { mean }\end{array}$ & Df & t-value & P-value & Remark \\
\hline L-arginine & Test & 90 & $36.29 \pm 48.15$ & 5.076 & 138 & -3.40 & 0.001 & SS \\
\hline & Control & 50 & $60.95 \pm 23.72$ & 3.35 & & & & \\
\hline
\end{tabular}

$\mathrm{N}=$ Number, $\mathrm{SD}=$ standard Deviation, $\mathrm{Df}=$ Degree of freedom, $\mathrm{SS}=$ Statistical significance.

Table 1: Results of mean score and serum L-arginine levels among pre-school children with malaria and non-malaria parasitized controls.

Table 2 shows the serum L-arginine levels among malaria parasitized children based on age. L-arginine was marginally lower among children in the 3 years age group $(22.93 \pm 15.49 \mu \mathrm{mol} / \mathrm{l})$ compared to children in the 4 years age group $(42.42 \pm 63.11 \mu \mathrm{mol} / \mathrm{l})$. The difference was however not statistically significant $(\mathrm{p}=0.11)$.

\begin{tabular}{|c|c|c|c|c|c|c|c|c|}
\hline Parameter & Age & $\mathbf{N}$ & Mean \pm SD & Std.Error mean & Df & t-value & p-value & Remark \\
\hline L-arginine & 3 & 29 & $22.93 \pm 15.49$ & 2.87 & 50 & -1.60 & 0.11 & NSS \\
\hline & 4 & 23 & $42.42 \pm 63.11$ & 13.16 & & & & \\
\hline L-arginine & 3 & 29 & $22.93 \pm 15.49$ & 2.88 & 65 & -1.95 & 0.05 & NSS \\
\hline & 5 & 38 & $42.77 \pm 53.12$ & 8.62 & & & & \\
\hline L-arginine & 4 & 23 & $42.42 \pm 63.11$ & 13.16 & 59 & -0.02 & 0.98 & NSS \\
\hline
\end{tabular}

Key: $\mathrm{N}=$ Number, $\mathrm{SD}=$ standard Deviation, $\mathrm{Df}=$ Degree of freedom, NSS =Non-Statistical significance.

Table 2: Serum L-arginine levels among malaria parasitized children based on age. 
Table 3 shows the serum L-arginine levels among malaria parasitized children based on gender. There were no significance differences in the mean L-arginine level among male children (36.64 $\pm 53.95 \mu \mathrm{mol} / \mathrm{l})$ compared to female children (35.73 $\pm 38.03 \mu \mathrm{mol} / \mathrm{l})$ $(\mathrm{p}=0.93)$.

\begin{tabular}{|c|c|c|c|c|c|c|c|c|}
\hline Parameter & Gender & $\mathbf{N}$ & Mean $\mathbf{\text { SD }}$ & Std. Error mean & Df & t-value & p-value & Remark \\
\hline L-arginine & Male & 55 & $36.64 \pm 53.95$ & 7.27 & 88 & 0.09 & 0.93 & NSS \\
\hline & Female & 35 & $35.73 \pm 38.03$ & 6.43 & & & & \\
\hline
\end{tabular}

Key: $\mathrm{N}=$ Number, $\mathrm{SD}=$ standard Deviation, $\mathrm{Df}=$ Degree of freedom, NSS =Non-Statistical significance

Table 3: Serum L-arginine levels among malaria parasitized children based on gender.

Table 4 shows the serum L-arginine levels among malaria parasitized children based on ethnicity. From the study, it was observed that there was no significance difference in the L-arginine level based on ethnicity $(p=0.59)$.

\begin{tabular}{|c|c|c|c|c|c|c|c|c|}
\hline Parameter & Ethnicity & $\mathbf{N}$ & Mean \pm SD & Std. Error Mean & Df & t-value & P-value & Remark \\
\hline \multirow{2}{*}{ L-arginine } & Hausa/Fulani & 69 & $29.75 \pm 28.71$ & 3.46 & 73 & 0.50 & 0.62 & NSS \\
\hline & Others & 6 & $23.80 \pm 14.46$ & 5.90 & & & & \\
\hline
\end{tabular}

Key: $\mathrm{N}=$ =Number, $\mathrm{SD}=$ standard Deviation, $\mathrm{Df}=$ Degree of freedom, $\mathrm{SS}=$ Statistical significance, NSS $=$ Non-Statistical significance.

Table 4: Serum L-arginine levels among malaria parasitized children based on ethnicity.

Table 5 shows the serum L-arginine levels among malaria parasitized children based on severity. Larginine was significantly lower among children with severe malaria $(15.65 \pm 7.52 \mu \mathrm{mol} / \mathrm{l})$ compared to those with uncomplicated malaria (55.76 $\pm 39.08 \mu \mathrm{mol} / \mathrm{l})$ $(p=0.000)$.

\begin{tabular}{|l|c|c|c|c|c|c|c|c|}
\hline Parameter & Malaria & N & Mean \pm SD & Std. Error Mean & Df & t-value & p-value & Remark \\
\hline L-arginine & Severe Malaria & 55 & $15.65 \pm 7.52$ & 1.01 & 88 & -7.42 & 0.000 & SS \\
\hline & Uncomplicated Malaria & 35 & $55.76 \pm 39.08$ & 6.61 & & & & \\
\hline
\end{tabular}

Key: $\mathrm{N}=$ Number, $\mathrm{SD}=$ standard Deviation, Df =Degree of freedom, SS= Statistical significance.

Table 5: Serum L-arginine levels among malaria parasitized children based on severity.

\section{Discussion}

Malaria is the most important tropical parasitic disease affecting about 247 million people each year, resulting in nearly a million deaths, mostly children under the age of five years [1]. Nearly $90 \%$ of these deaths occur in Africa South of the Sahara where a child dies every 30 seconds [2]. Severe malaria due to Plasmodium falciparum claims 0.6-1.2 million lives annually, $86 \%$ of whom are children in sub-Saharan Africa [29]. Despite the use of highly effective antimalarial medications, 10-30\% patients with severe malaria will die [30], highlighting the need for new adjunctive therapy. This study investigated the level of l-arginine among pre-school children with malaria (subjects) and non-parasitized malaria children (control) in UDUTH Sokoto, Nigeria.
In this study, we observed that the l-arginine levels among children with malaria was significantly lower compared to non-parasitized controls $(p=0.001)$. In severe malaria patients, supplement with L-Arginine has been shown to improve NO bioavailability and reverse endothelial dysfunction [31]. L-Arginine infusion has proven to be safe in malaria patients and is currently undergoing clinical trial for preventing endothelial dysfunction during severe malaria [32]. As L-Arginine is the substrate of NO synthase (NOS) for the production of NO, hypoargininemia can lead to impaired systemic NO production [11]. The effect of LArginine supplement is mostly likely due to enhanced production of NO, and the importance of NO in host defense against a variety of pathogens has been well documented [33]. The reason for the low level of 1arginine among malaria parasitized children in this 
study is not far-fetched. In malaria parasite infestation, RBC are altered and undergo haemolysis, and these RBC bind to endothelial cells resulting in interference with blood flow [34]. The malaria -related haemolysis is associated with release of RBC cytoplasmic contents such as haemoglobin, lactate dehydrogenase and arginase into the plasma. Plasma free haemoglobin reduces NO bioavailability as its binds NO and quenches/scavenges its bioactivity [35]. Plasma arginase diminishes arginine levels, and thus may reduce production of NO. L-arginine and NO are protective against severe malaria parasites which causes organ/tissue damage. Severe malaria is associated with low L-arginine and by extension nitric oxide production. Hypoargininemia is likely to result in sub-optimal monocyte, endothelial function, and tissue NO production because the intracellular NOS activity is critically dependent on adequate circulating L-arginine. Moreover, hypoargininemia may enhance the deleterious oxidative stress.

The use of l-arginine supplementation in malaria infection has been suggested [36]. Increase in plasma levels of l-arginine and within and above the normal range will be expected to increase NO production, restores plasma arginine levels to normal, increases systemic and exhaled NO production, reduces oxidant stress, and improves a number of physiological measures of relevance to malaria (including vascular endothelial function and physiological dead space). Larginine concentrations fall in the settings of inflammation, haemolysis and malaria infection, and Larginine levels vary inversely with malaria disease severity. It is hypothesized that the low bioavailability of arginine for NO synthesis contributes to pathogenesis.

Previous studies have proposed that L-arginine should be used as a potential adjunctive therapy in severe malaria $[24,31,37,38]$. The rationale for this is based on previous findings in severe malaria of impaired NO production [31], hypoargininemia [24], near-universal impairment of NO-dependent endothelial function [31] and a close association between the improvement in endothelial function and recovery of plasma L-arginine concentrations after treatment of severe malaria [32]. Previous report has demonstrated that L-arginine infusion is able to improve NO bioavailability and endothelial function in patients with moderately severe malaria [31], suggesting the potential for a similar effect if used as adjunctive therapy in severe malaria. Possible causes of hypoargininemia in severe malaria include decreased larginine synthesis and increased catabolism caused by the increased activity of plasma arginase [39] and/or cytokine-inducible arginase in endothelial cells or immune cells $[40,41]$. As well as impairing endothelial function, hypoargininemia and NO deficiency may contribute to pathology in severe malaria through other mechanisms. NO inhibits platelet activation [42], and deficiency may therefore exacerbate platelet-mediated processes linked to the microvascular pathology of severe malaria, including endothelial cell activation [43] and apoptosis and platelet-mediated clumping [44].

In this study, we observed an inverse relationship between malaria severity and l-arginine level. The larginine level was significantly lower among preschool children with severe malaria compared to those with uncomplicated P-falciparum malaria. Our finding is consistent with the finding in a previous report among plasmodium parasitized Tanzania children which indicated that African children with severe malaria have impaired production of NO and that reduced bioavailability of NO contributes to the pathogenesis of severe malaria [45] and low plasma arginine levels [24], the substrate for NO synthesis. Treatment of Indonesian adults with severe malaria with intravenous l-arginine increased levels of exhaled NO, and reversed malariaassociated endothelial dysfunction [31]. Pathways of endothelial activation and dysfunction are reflected in experimental models of severe malaria, where inhaled NO enhances endothelial integrity, reduces parasite accumulation in the brain vasculature, and improves survival $[46,47]$. L-arginine levels are low in patients with CM, moderately low in patients with uncomplicated malaria and normal in healthy controls [24]. Hypoargininemia caused by arginase release from haemolyzed erythrocytes is significantly associated with fatal outcome in patients with CM [24]. Exogenous L-arginine rescues reactive hyperemia-peripheral artery tonometry, a noninvasive measurement of vascular NO production, in patients with severe malaria [31]. Asymmetric dimethylarginine levels are higher in patients with severe malaria than in those with moderately severe malaria and each micromolar increase of this endogenous inhibitor of NOS conversion of L-arginine to NO is associated with an 18-fold increase in mortality [48].

Severe malaria is characterized by hypoargininemia, haemolysis, decreased pulmonary NO, and impairment of endothelial function, with each potentially contributing to its pathogenesis. Given its central role in malaria pathophysiology, the endothelium may be an important therapeutic target for adjunctive treatment. Short-term parenteral l-arginine therapy is safe in MSM and increases pulmonary NO production. Its efficacy in improving endothelial function suggests that hypoargininemia and decreased NO bioavailability contribute to endothelial dysfunction. Our work provides firm proof of concept to warrant clinical trials 
of adjunctive agents such as l-arginine to improve endothelial NO bioavailability in severe malaria. Finding from this study is justification to possibly provide nitric oxide producing l-arginine supplement as adjunctive therapy along with malaria chemotherapeutics in the management of pre-school children with P-falciparum malaria. Previous report [31] indicate that endothelial dysfunction in malaria is nearly universal in severe disease, is reversible with l-arginine, and likely contributes to its pathogenesis. Clinical trials in SM of adjunctive agents to improve endothelial NO bioavailability, including l-arginine, are warranted [37].

Possible causes of hypoargininaemia in SM include decreased l-arginine synthesis and increased catabolism caused by the increased activity of plasma arginase [49] and/or cytokine-inducible arginase in endothelial cells or immune cells [50]. As well as impairing endothelial function, hypoargininemia and NO deficiency may contribute to pathology in SM through other mechanisms. NO inhibits platelet activation [51], and deficiency may therefore exacerbate platelet-mediated processes linked to the microvascular pathology of SM, including endothelial cell activation [42,43] and apoptosis and platelet-mediated clumping. Because NO is also a determinant of red cell deformability, deficiency of L-arginine and by extension NO may exacerbate the impaired red cell deformability found in $\mathrm{SM}$, further compromising microvascular perfusion.

In this study, we observed that there was no significant difference in the serum L-arginine level based on age $(p>0.05)$. This finding is at variance with a previous report which suggests that hypoargininemia is characteristic of severe malaria across different age group.

We observed that there was no significant difference in the L-arginine level based on the gender of the malaria parasitized subjects $(p=0.93)$. The reason for non-significance is unknown. Previous report indicates that L-arginine level and by extension nitric oxide production is gender related. The reason for the differences in the levels of l-arginine based on gender is unknown. However hormonal differences between male and female gender may play a significant role.

We observed that there was no statistically significant difference in the serum L-arginine level based on ethnicity. The ethnic/racial impact on health has been receiving considerable attention in recent years. The mechanisms underlying racial disparities may be multifactorial and involve genetic factors, socioeconomic status, nutritional status, psychosocial stressors/risks, and other environmental factors.

\section{Conclusion}

In conclusion, the findings from this study confirmed that the level of l-arginine among pre-school children with malaria is significantly lower compared with that of uncomplicated malaria and non-parasitized controls. It may be necessary to routine provide nitric oxide generating l-arginine supplementation as adjunctive agents along with antimalarial chemotherapeutics in the treatment of malaria parasitized children. There is need to routinely monitor the nitric oxide levels of malaria parasitized children in Sokoto, North Western Nigeria in particular and Nigeria in general. Public awareness and education campaign should be carried out by the State and Federal government to educate parents on the need to provide a balance diet rich in 1arginine for their wards.

\section{References}

1. WHO (2008) World Health Organization, Geneva. WHO World Malaria Report.

2. WHO (2005a) Making every mother and child count. World Health Organization, Geneva. The World Health Report.

3. WHO (2005b) World Health Organization, Geneva. WHO World Malaria Report.

4. Brero A, Ramella R, Fitou A, Dati C, Alloatti G, et al. (2010) Neuregulin-1beta1 rapidly modulates nitric oxide synthesis and calcium handling in rat cardiomyocytes. Cardiovas Res 88(3): 443-452.

5. Jung HJ, Yang MZ, Kwon KH, Yenari MA, Choi YJ, et al. (2010) Endogenous agmatine inhibits cerebral vascular matrix metalloproteinases expression by regulating activating transcription factor 3 and endothelial nitric oxide synthesis. Curr Neurovas Res 7(3): 201-212.

6. Boucher JL, Moali C, Tenu JP (1999) Nitric oxide biosynthesis, nitric oxide synthase inhibitors and arginase competition for L-arginine utilization. Cell Mol Life Sci 55(8-9): 1015-1028.

7. Van Eijk AM, Hill J, Noor AM, Snow RW, ter Kuile FO (2015) Prevalence of malaria infection in pregnant women compared with children for tracking malaria transmission in sub-Saharan Africa: a systematic review and meta-analysis. Lancet Glob Health 3(10): 617-628.

8. FMH (2005b) Malaria Desk Situation Analysis Federal Ministry of Health. Publication of the FMH, Nigeria, FGN Publication: 27. 
9. John CC, Kutamba E, Mugarura K, Opoka RO (2010) Adjunctive therapy for cerebral Malaria and other severe forms of Plasmodium falciparum malaria. Expert Rev Anti Infect Ther 8(9): 997-1008.

10. Weinberg J (1998) Nitric oxide production and nitric oxide synthase type 2 expression by human mononuclear phagocytes: a review. Mol Med 4(9): 557-591.

11. Omodeo-Sale F, Cortelezzi L, Vommaro Z, Scaccabarozzi D, Dondorp AM (2010) Dysregulation of L-arginine metabolism and bioavailability associated to free plasma heme. Am J Physiol Cell Physiol 299(1): 148-154.

12. World Health Organization, Communicable Diseases Cluster Severe falciparum malaria (2000) Translation of the Royal Society of Tropical Medicine and Hygiene. 94(1): 1-90.

13. Aikawa M, Iseki M, Barnwell JW, Taylor D, Oo MM, et al. (1990) The pathology of human cerebral malaria. Am J Trop Med Hyg 43(2): 30-37.

14. Turner G (1997) Cerebral malaria. Brain Pathology $7(1): 569-582$.

15. Idro R, Jenkins NE, Newton CR (2005) Pathogenesis, clinical features, and neurological outcome of cerebral malaria. Lancet Neurol 4(12): 827-840.

16. Brown H, Turner $G$, Rogerson $S$, Tembo $M$, Mwenechanya J, et al. (1999) Cytokine expression in the brain in human cerebral malaria. J Infect Dis 180(5): 1742-1746.

17. Kwiatkowski D, Hill AV, Sambou I, Twumasi P, Castracane J, et al. (1990) TNF concentration in fatal cerebral, non-fatal cerebral, and uncomplicated Plasmodium falciparum malaria. Lancet 336(8725): 1201-1204.

18. Brown $H$, Rogerson $S$, Taylor $T$, Tembo $M$, Mwenechanya J, et al. (2001) Blood-brain barrier function in cerebral malaria in Malawian children. Am J Trop Med Hyg 64(3-4): 207-213.

19. Brown HC, Chau TT, Mai NT, Day NP, Sinh DX, et al. (2000) Blood-brain barrier function in cerebral malaria and CNS infections in Vietnam. Neurology 55(1): 104-111.

20. Looareesuwan S, Wilairatana P, Krishna S, Kendall B, Vannaphan S, et al. (1995) Magnetic resonance imaging of the brain in patients with cerebral malaria. Clin Infect Dis 21(2): 300-309.
21. Newton CR, Peshu N, Kendall B, Kirkham FJ, Sowunmi A, et al. (1994) Brain swelling and ischaemia in Kenyans with cerebral malaria. Arch Dis Child 70(4): 281-287.

22. Percário S, Moreira DR, Gomes BA, Ferreira ME, Gonçalves ACM, et al. (2012) Oxidative stress in malaria. Int J Mol Sci 13(12): 16346-16372.

23. McDevitt MA, Xie J, Gordeuk V, Bucala R (2004) The anemia of malaria infection: role of inflammatory cytokines. Curr Haematol Rep 3(2): 97-106.

24. Lopansri BK, Anstey NM, Weinberg JB, Stoddard GJ, Hobbs MR, et al. (2003) Low plasma arginine concentrations in children with cerebral malaria and decreased nitric oxide production. Lancet 361(9358): 676-678.

25. Boger RH, Bode-Boger SM (2001) The clinical pharmacology of L-arginine. Annu Rev Pharmacol Toxicol 41: 79-99.

26. NPC/FRN (2007) Nigeria Population Commission, Federal Republic of Nigeria. Special FGN Gazette No. 23 on the 2006 Population Census.

27. MOI (2008) Ministry of Information, Sokoto, Nigeria. Diary: 1 -2.

28. Udo RK, Mamman AB (1993) Nigeria: Giant in the tropics. State Surveys: 435-446.

29. Murray CJ, Rosenfeld LC, Lim SS, Andrews KG, Foreman KJ, et al. (2012) Global malaria mortality between 1980 and 2010: a systematic analysis. Lancet 379(9814): 413-431.

30. Kyu HH, Fernandez E (2009) Artemisinin derivatives versus quinine for cerebral malaria in African children: a systematic review. Bull World Health Organ 87(12): 896-904.

31. Yeo TW, Lampah DA, Gitawati R, Tjitra E, Kenangalem E, et al. (2007) Impaired nitric oxide bioavailability and L-arginine-reversible endothelial dysfunction in adults with falciparum malaria. J Exp Med 204(11): 2693-2704.

32. Yeo TW, Lampah DA, Gitawati R, Tjitra E, Kenangalem E (2008) Recovery of endothelial function in severe falciparum malaria: association with improvement in plasma L-arginine and blood lactate concentrations. J Infec Dis 198(4): 602-608. 
33. Peranzoni E, Marigo I, Dolcetti L, Ugel S, Sonda N, et al. (2007) Role of arginine metabolism in immunity and immunopathology. Immunobiology 212(9-10): 795-812.

34. Rowe JA, Claessens A, Corrigan RA, Arman M (2009) Adhesion of Plasmodium falciparum-infected erythrocytes to human cells: molecular mechanisms and therapeutic implications. Expert Rev Mol Med 11: e16.

35. Schaer DJ, Buehler PW, Alayash AI, Belcher JD, Vercellotti GM (2013) Hemolysis and free hemoglobin revisited: exploring hemoglobin and hemin scavengers as a novel class of therapeutic proteins. Blood 121(8): 1276-1284.

36. Mohanty S, Patel DK, Pati SS, Mishra SK (2006) Adjuvant therapy in cerebral malaria. Indian J Med Res 124(3): 245-260.

37. Pasvol G (2005) Management of severe malaria: interventions and controversies. Infect Dis Clin North Am 19(1): 211-240.

38. Dondorp A, Nosten F, Stepniewska K, Day N, White N (2005) Artesunate versus quinine for treatment of severe falciparum malaria: a randomised trial. Lancet 366(9487): 717-725.

39. Argaman Z, Young VR, Noviski V, Castillo-Rosas L, Lu XM, et al. (2003) Arginine and nitric oxide metabolism in critically ill septic pediatric patients. Crit Care Med 31: 591-597.

40. Bachetti T, Comini L, Francolini G, Bastianon D, Valetti B, et al. (2004) Arginase pathway in human endothelial cells in pathophysiological conditions. J Mol Cell Cardiol 37(2): 515-523.

41. Hesse M, Modolell M, La Flamme AC, Schito M, Fuentes JM, et al. (2001). Differential regulation of nitric oxide synthase- 2 and arginase- 1 by type 1/type 2 cytokines in vivo: granulomatous pathology is shaped by the pattern of L-arginine metabolism. J Immunol 167(11): 6533-6544.

42. Freedman JE, Loscalzo J (2003) Nitric oxide and its relationship to thrombotic disorders. J Thromb Haemost 1(6): 1183-1188.

43. Grau GE, Mackenzie CD, Carr RA, Redard M, Pizzolato G, et al. (2003) Platelet accumulation in brain microvessels in fatal pediatric cerebral malaria. J Infect Dis 187(3): 461-466.

44. Pain A, Ferguson DJ, Kai O, Urban BC, Lowe B, et al. (2001) Platelet-mediated clumping of Plasmodium falciparum-infected erythrocytes is a common adhesive phenotype and is associated with severe malaria. Proc Natl Acad Sci USA 98(4): 1805-1810.

45. Anstey NM, Weinberg JB, Hassanali MY, Mwaikambo ED, Manyenga D, et al. (1996) Nitric oxide in Tanzanian children with malaria: inverse relationship between malaria severity and nitric oxide production/nitric oxide synthase type 2 expression. J Exp Med 184(2): 557-567.

46. Serghides L, Kim H, Lu Z, Kain DC, Miller C, et al. (2011) Inhaled nitric oxide reduces endothelial activation and parasite accumulation in the brain, and enhances survival in experimental cerebral malaria. PLoS One 6: e27714.

47. Gramaglia I, Sobolewski P, Meays D, Contreras R, Nolan JP, et al. (2006) Low nitric oxide bioavailability contributes to the genesis of experimental cerebral malaria. Nat Med 12(12): 1417-1422.

48. Yeo TW, Lampah DA, Tjitra E, Gitawati R, Darcy CJ, et al. (2010) Increased asymmetric dimethylarginine in severe falciparum malaria. Association with impaired nitric oxide bioavailability and fatal outcome. PLoS Pathog 6(4): e1000868.

49. Bor-Kucukatay M, Wenby RB, Meiselman HJ, Baskurt OK (2003) Effects of nitric oxide on red blood cell deformability. Am J Physiol Heart Circ Physiol 284(5): 1577-1584.

50. Dondorp AM, Pongponratn E, White NJ (2004) Reduced microcirculatory flow in severe falciparum malaria: pathophysiology and electron-microscopic pathology. Acta Trop 89(3): 309-317.

51. Mata-Greenwood E, Chen D (2008) Racial Differences in Nitric Oxide-Dependent Vasorelaxation. Reprod Sci 15(1): 9-25.

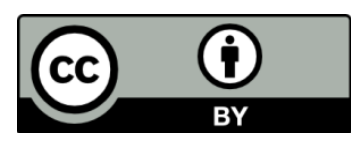

